\title{
A study assessing the post operative outcome in patients of acoustic schwannoma operated through retrosigmoid approach at tertiary care institutions- An experience of one year
}

\author{
Sourabh Dixit', Manpreet Singh Banga', Suniti Kumar Saha², Kaushik Roy ${ }^{3}$, \\ Partha Ghosh', Sandeep BV' \\ ${ }^{1}$ Post Doctoral Trianee, ${ }^{2}$ Professor and Head, ${ }^{3}$ Associate Professor, Department of Neurosurgery, NRS Medical \\ College, Kolkata
}

A B S TR A C T

Background: The most common tumors of the cerebellopontine angle (CPA) is Acoustic schwannoma (80-90\%). Cerebellopontine Angle tumors may be asymptomatic or may present with symptoms which could be due to tumor mass or due to raised intracranial pressure (ICP). The Suboccipital retrosigmoid approach has become an increasingly popular method of excising CPA tumors. Aims and Objectives: To assess the post operative outcome and incidence of post operative complications in patients operated for CPA tumor through retrosigmoid approach. Materials and Methods: The present "Prospective study" was carried out in 10 patients admitted to the Department Of Neurosurgery, NRSMCH, Kolkata with a clinicoradiological diagnosis of CPA tumor and were operated between Feruary 2016 to March 2017. Results: The maximum number of cases i.e $38.46 \%(n=5)$ were of 31 to 40 years of age. The male to female ratio was $0.3: 1$. The most common symptom were ipsilateral sensorineural hearing loss (SNHL) and tinnitus, headache associated with papilloedema. The gross total resection was done in $84.61 \%$ cases \& subtotal done in $15.38 \%$ cases.CSF leakage was present in $23.07 \%$ cases. Facial Nerve function of grade 1 was present in $53.84 \%$. Out of all cases Grade 2 and grade 5 function was present in 1 patient. Grade $3 \&$ Grade 4 dysfunction were present in 2 patients. Transient lower cranial nerve paresis was present in $46.15 \%$. One patient expired in postoperatively. Conclusion: Ventriculo-peritoneal shunting followed by excision of tumor by retrosigmoid approach can bring good neurological outcome in patients with CPA tumors.

Key words: Acoustic schwannoma, Retrosigmoid approach, Venticulo-peritoneal shunt
http://nepjol.info/index.php/AJMS DOI: $10.3126 /$ ajms.v8i4.17260 E-ISSN: 2091-0576 P-ISSN: 2467-9100

\section{INTRODUCTION}

The Acoustic schwannoma is the most common tumors of the cerebellopontine angle (CPA) (80-90\%) followed by Meningioma (5-10\%), Ectodermal inclusion tumours, Epidermoid and Dermoid (5-7\%). Other tumors of the CPA include primary cholesteatoma, and other cranial nerve schwannoma (V, VII, IX, X), arachnoid cysts etc. These tumors may be asymptomatic or may present with a constellation of symptoms which could be due to tumor mass and involvement of the adjacent cranial nerves, cerebellum and brainstem. Furthermore there could be associated signs and symptoms due to raised intracranial pressure (ICP) due to regional brainstem compression and hydrocephalus. The most common symptoms of are unilateral sensorineural hearing loss (96\%), unsteadiness $(77 \%)$, tinnitus $(71 \%)$, headache $(29 \%)$, mastoid pain or otalgia (28\%), facial numbness $(7 \%)$ and diplopia $(7 \%) .{ }^{1}$

The options available for management includes Observation, Surgery, Stereotactic Radiosurgery, Fractionated radiotherapy. Some patients might also be candidates for a combination 
of these therapies. ${ }^{2}$ The ideal treatment is total excision of tumor. Surgery is indicated for small, medium and large size tumors. ${ }^{3}$ Several approaches have been advocated and various techniques are available for the resection of CPA tumors, including translabyrinthine, retrosigmoid, suboccipital, retrolabyrinthine, transcochlear, transotic and middle fossa approaches. ${ }^{1-4}$ Surgery improves quality of life in patients but each approach is associated with post operative complications like mortality, haematoma, pneumocephalus, cranial neuropathies, cerebellar dysfunction, brainstem complications, infections, CSF leaks etc. ${ }^{1-4}$

The Suboccipital retrosigmoid approach has become an increasingly popular method of excising CPA tumors. ${ }^{5}$ Advantages of this approach include a low complication rate, particularly with regard to facial nerve function, and total tumor removal in the vast majority of cases. Moreover, the technique is safe and effective, even with the largest of tumors. ${ }^{1,3,5}$

The present study was undertaken to evaluate the post surgery outcome in patients admitted and operated for Acoustic schwannoma through retrosigmoid approach in NRSMCH.

\section{AIMS AND OBJECTIVES}

1. To assess the post operative outcome in patients of acoustic schwannoma operated through retrosigmoid approach.

2. To assess the incidence of post operative complications in patients.

\section{MATERIALS AND METHODS}

The present "Prospective study" was carried out in 13 patients admitted to the Department Of Neurosurgery, NRSMCH, Kolkata with a clinicoradiological diagnosis of acoustic schwannoma and were operated between Feb 2016 to March 2017.

\section{Surgery}

The surgical management was done in two stages;- in first stage the patients were subjected to venticulo-peritonial shunting to relieve the raised intra cranial pressure (ICP) and in second stage the excision of tumor was done through Retrosigmoid approach. After the management of post operative period the patient was discharged with advice for regular follow up in out patient department.

\section{Inclusion Criteria}

Patients admitted with clinicoradiological diagnosis of acoustic schwannoma.
Patients operated by retrosigmoid approach.

\section{Exclusion criteria}

Patients who had been operated by other approaches.

The various parameters that were used in the study were size of tumor. Tumors were categorized according to their cerebellopontine angle dimensions as Group 1 $(<2 \mathrm{~cm})$, Group $2(2-3.9 \mathrm{~cm})$, and Group $3(>$ or $=4 \mathrm{~cm}),{ }^{6}$ preoperative neurological deficits, extent of cranial nerve involvements, functional status of patients in preoperative and postoperative periods, presence of signs of raised ICP etc and postoperative complications were recorded in immediate and delayed post operative periods. Facial nerves functioning in post operative periods were measured by House Brackmann Facial nerve Grading system. ${ }^{7}$ A post operative follow up of the patient was done for 6 months. Post operative lower cranial nerve paresis was assessed by presence or absence of Gag reflex and deglutination difficulty. The data of relevant information was collected and tabulated and systematically analysed.

\section{RESULTS}

In the present series the following outcomes were obtained:-

\section{Age}

Regarding age incidence (Table 1 ) the maximum number of cases i.e $38.46 \%(n=5)$ were of 31 to 40 years of age. This was followed by $30.76 \%$ incidence in $21-30 \mathrm{yrs}$ age group. This was followed by incidence of $23.03 \%$ in age groups of $41-50 \mathrm{yrs}$ and $7.69 \%$ in $61-70$ years. The average age of presentation was 37.23 years and Standard deviation (SD) was 11.410.

\section{Sex}

Regarding the sex (Table 2) the maximum number of patients i.e $76.92 \%(n=10)$ were Female. The number of male patients was 3 i.e $23.07 \%$ of total cases. The male to female ratio was 0.3:1.

\section{Size of tumour}

Maximum number of cases (Table 3) presented to us with tumor size between $2 \mathrm{~cm}-3.9 \mathrm{~cm},($ Group 2) i.e 53.84\%

\begin{tabular}{lcc}
\multicolumn{3}{l}{ Table 1: Age wise distribution of patients } \\
\hline Age interval & Males & Females \\
\hline 21years to 30 years & 1 & 3 \\
31 years to 40 years & 1 & 4 \\
41 years to 50 years & 0 & 3 \\
51 years to 60 years & 0 & 0 \\
$>60$ years & 1 & 0 \\
\hline
\end{tabular}


$(\mathrm{n}=7)$. This was followed by with tumor size $>4$ (Group 3) i.e $30.77 \%(n=4)$. This was followed by with tumor size $<2 \mathrm{cms}$ (Group 1) i.e 15.38\% $(\mathrm{n}=2)$ ). The average size of tumor was $3.21 \mathrm{~cm}$ and Standard deviation (SD) was 0.990 .

\section{Preoperative signs \& symptoms}

The most common symptom (Table 4) that was present among the patients was ipsilateral sensorineural hearing loss (SNHL) and tinnitus which was present in all 13 patients $(100 \%)$. Out of these grade 4 SNHL was present in $53.84 \%$ patients $(n=7)$, followed by grade 3 SNHL was present in $46.15 \%$ patients $(n=6)$. Headache associated with sign of raised intracranial pressure in form of papilloedema was also present in all 13 patients $(n=13)$. Among the Group 1 , the $2(15.38 \%)$ patients had grade 1 papilloedema. Among Group 2, the grade 1 and grade 2 papilloedema was found in 2 patients each $(15.38 \%)$ and grade 3 pailloedema was found in 3 patients $(23.07 \%)$. Among Group 3, the grade 3 papilloedema was found in 1 patient $(7.69 \%)$ and grade 4 pailloedema was found in 3 patients $(23.07 \%)$.

Vestibular symptoms were present in $53.84 \%(n=7)$ of patients. Vestibular symptoms were absent in group 1 patients. In group 2 Vestibular symptoms were present in 3 patients $(23.07 \%)$ and in group 3 Vestibular symptoms were present in 4 patients $(30.76 \%)$.

Facial paresis was present in $76.92 \%(\mathrm{n}=10)$ of patients. Facial weakness was absent in Group 1 patients. In group 2 Facial paresis was present in 6 patients $(46.15 \%, n=3)$ and in group 3 Facial weakness was present in 4 patients $(30.76 \%, \mathrm{n}=6)$.

Trigeminal involvement was present in 53.84\% $(\mathrm{n}=7$ ) of patients. Trigeminal involvement was absent in

\begin{tabular}{lc} 
Table 2: Sex distribution among patient & \\
\hline Sex & Number \\
\hline Male & 3 \\
Female & 10 \\
Ratio & $0.3: 1$ \\
\hline
\end{tabular}

\section{Table 3: Size wise distribution of tumour}

\begin{tabular}{lcc}
\hline Size of tumour & Males & Females \\
\hline Group 1 $(<2 \mathrm{~cm})$ & 2 & 0 \\
Group 2 $(2-3.9 \mathrm{~cm})$ & 0 & 7 \\
Group 3 $(>4 \mathrm{~cm})$ & 1 & 3 \\
\hline
\end{tabular}

Group 1 patients. Trigeminal involvement in group 2 was present in 4 patients $(30.76 \%)$ and in group 3 trigeminal involvement was present in 3 patients (23.07\%). Among the trigeminal involvement the most common symptom was facial numbness on ipsilateral side and absence of corneal reflex.

Lower cranial nerve involvement was present in 38.46\% $(n=5)$ of patients. Lower cranial nerve involvement was absent in Group 1 patients. Lower cranial nerve involvement in group 2 was present in 2 patients $(15.38 \%$ ) and in group 3 lower cranial nerve involvement was present in 3 patients (23.07\%). Among the lower cranial nerve involvement the most common finding was diminished gag reflex.

Cerebellar signs were present in $76.92 \%(n=10)$ of patients. Cerebellar involvement was absent in Group 1 patients. Cerebellar involvement in group 2 was present in 6 patients $(46.15 \%)$ and in group 3 trigeminal involvement was present in 4 patients $(30.76 \%)$. Among the cerebellar the most common symptom was gait unsteadiness with difficulty in walking in form of swaying.

Pyramidal tract signs were present in 30.76\% $(n=4)$ of patients. Pyramidal tract involvement was absent in Group 1 and group 2 patients. Long tract involvement was seen in group 3 in 4 patients.

\section{Postoperative outcomes}

The gross total resection (Table 5) was done in $84.61 \%(n=11)$ cases and subtotal done in 15.38\% $(\mathrm{n}=2)$ cases. In Group 1 the gross total removal of tumor was done in all the 2 patients. In group 2 subtotal resection was done in $1(7.69 \%$, ) patients and gross total resection was done in $6(46.15 \%)$ patients. In group 3 subtotal resection was done in $1(7.69 \%)$ patients and gross total resection was done in $3(23.07 \%)$ patients.

CSF leakage was present in post operative in $23.07 \%$ cases. Out of these 1 case were in group 2 and 2 cases were from group 3.

Residual tumor was present in 2(15.38\%) patients. Out of these 1 case belonged to Group 2 and 1 case belonged to group 3 .

Post operative facial nerve functioning was assessed using House and Brackmann grading. ${ }^{7}$ Facial Nerve function of

\section{Table 4: Preoperative signs and symptoms in patients}

\begin{tabular}{|c|c|c|c|c|c|c|c|c|c|}
\hline Size of tumor & SNHL & Tinnitus & Papilloedema & $\begin{array}{l}\text { Vestibular } \\
\text { symptoms }\end{array}$ & $\begin{array}{l}\text { Facial } \\
\text { paresis }\end{array}$ & $\begin{array}{l}\text { Trigeminal } \\
\text { paresis }\end{array}$ & $\begin{array}{c}\text { Lower cranial } \\
\text { nerves }\end{array}$ & $\begin{array}{l}\text { Cerbellar } \\
\text { signs }\end{array}$ & $\begin{array}{c}\text { Pyramidal } \\
\text { sign }\end{array}$ \\
\hline Group 1(<2 cm) & 2 & 2 & 2 & 0 & 0 & 0 & 0 & 0 & 0 \\
\hline Group $2(2-3.9 \mathrm{~cm})$ & 7 & 7 & 7 & 3 & 6 & 4 & 2 & 6 & 0 \\
\hline Group $3(>4 \mathrm{~cm})$ & 4 & 4 & 4 & 4 & 4 & 3 & 3 & 4 & 4 \\
\hline
\end{tabular}




\begin{tabular}{|c|c|c|c|c|c|c|}
\hline Size of tumor & $\begin{array}{l}\text { Resection of tumor } \\
\text { gross total/subtotal }\end{array}$ & CSF leakage & $\begin{array}{l}\text { Facial function } \\
\text { (HBS) }\end{array}$ & $\begin{array}{l}\text { Residual } \\
\text { tumor }\end{array}$ & $\begin{array}{l}\text { Lower cranial } \\
\text { nerves involvement }\end{array}$ & Mortality \\
\hline Group 1(<2 cm) & 2 Gross total - 2 & 0 & Grade 1=2 & 0 & 0 & 0 \\
\hline Group $2(2-3.9 \mathrm{~cm})$ & $\begin{array}{l}7 \text { Gross total }-6 \\
\text { Subtotal-1 }\end{array}$ & 1 & $\begin{array}{l}\text { Grade 2=1 } \\
\text { Grade 3=1 }\end{array}$ & 1 & 2 & 0 \\
\hline Group 3 (>4 cm) & $\begin{array}{l}4 \text { Gross total-3 } \\
\text { Subtotal- } 1\end{array}$ & 2 & $\begin{array}{l}\text { Grade } 3=1 \\
\text { Grade } 4=2 \\
\text { Grade } 5=1\end{array}$ & 1 & 4 & 1 \\
\hline
\end{tabular}

grade 1 was present in $53.84 \%$. Out of all cases grade 2 and grade 5 functions were present in 1 patient. Grade 3 and grade 4 dysfunction were present in 2 patients. In post operative follow up total 4 patient had symptomatic improvement. Transient lower cranial nerve paresis was present in $46.15 \%$.

\section{DISCUSSION}

Age \& sex

The maximum number of cases i.e $38.46 \%(n=5)$ were of 31 to 40 years of age. The average age of presentation was 37.23 years and standard deviation (SD) was 11.410. Memari F et al had mean age of 49 years in their series. ${ }^{1}$ There was a female predominance in the present series but in series in series of Memari $\mathrm{F}_{\text {et }} \mathrm{al}^{1}$ there was male predominance.

\section{Size of tumors}

Maximum number of case had tumor size between $2 \mathrm{~cm}-3.9 \mathrm{~cm}$ i.e $53.84 \%(\mathrm{n}=7)$. The average size of tumor was $3.21 \mathrm{~cm}$ and standard deviation (SD) was 0.990 . Memari $\mathrm{F}$ et $\mathrm{al}^{1}$ had observed the mean tumor size was $24 \mathrm{~mm}$, ranging from $<15 \mathrm{~mm}$ to $>35 \mathrm{~mm}$.

\section{Preoperative signs \& symptoms}

The most common symptom was ipsilateral sensorineural hearing loss (SNHL) and tinnitus which was present in all 13 patients $(100 \%)$. Headache associated with sign of raised intracranial pressure in form of papilloedema was also present in all 13 patients $(n=13)$. Vestibular symptoms were present in $53.84 \%(\mathrm{n}=7)$ of patients. Facial paresis was present in $76.92 \%(n=10)$ of patients. Trigeminal involvement was present in $53.84 \%(n=7)$ of patients. Lower cranial nerve involvement was present in $38.46 \%$ $(n=5)$ of patients. Cerebellar signs were present in $76.92 \%$ $(n=10)$ of patients. Pyramidal tract signs were present in $30.76 \%(\mathrm{n}=4)$ of patients.

Memari $\mathrm{F}$ et $\mathrm{al}^{1}$ had observed that Forty-seven patients $(94 \%)$ presented with tinnitus, and vertigo was present in 30 patients $(60 \%)$. The preoperative pure tone average was $60 \mathrm{db}$. Fourteen patients $(28 \%)$ had a pure tone average $>50 \mathrm{db}$ and a word recognition score $>60 \%$. Seven patients had cranial nerve 7 paresis preoperatively (maximum House Brackmann score, 4). Cranial nerve 5 involvement was present in four patients preoperatively, all of whom had tumor size $>30 \mathrm{~mm}$. Two patients had cranial nerve X, XI, and XII involvement preoperatively and two patients had hemiplasia preoperatively. M. Javad Mirzayan et $\mathrm{al}^{8}$ had also obtained similar findings with Hypacusis/Deafness 70\% (12) Tinnitus 30\% (6)Vertigo 15\% (3) Cerebellar symptoms 10\% (2) Facial palsy 10\% (2)Trigeminal hypesthesia 5\% (1) Symptoms of increased ICP 15\% (3).

In the present series all patients had underwent preoperative ventriculoperitoneal shunting to relieve the raised ICP Followed by excision of tumour through retrosimid approach. Firm tumours adhered to brain stem were considered for subtotal excision. ${ }^{9}$ The gross total resection was done in $84.61 \%(n=11)$ cases \& subtotal done in $15.38 \%(n=2)$ cases.

Jain $\mathrm{VK}$ et $\mathrm{al}^{9}$ also advocated preoperative shunting in patients presenting with features of raised ICP. The incidence of preoperative shunting was as high as 66\% in the series reported by Ramamurthy. ${ }^{4}$

Gerganov VM et al ${ }^{10}$ recommended placement of external ventricular drainage (EVD) or VP shunt prior to surgery as surgery in patients with hydrocephalus and increased ICP is presumably more challenging, and related to worse outcome or higher complication rates. They found that the general and functional outcome in patients with primary VS removal is independent of the presence of hydrocephalus.

The retrosigmoid approach has been accepted as a popular approach for excision of small, medium and large size CP angle tumors. Merari $\mathrm{F}$ et $\mathrm{al}^{1}$ in their series had operated $28 \%$ cases by retrosigmiod approach and had low complication rates. Gormley and Sekhar et $\mathrm{al}^{6}$ used the combined transpetrosal and retrosigmoid approach for tumors greater or more than $4 \mathrm{~cm}$ in the cerebello-pontine angle, especially when they extend up to the tentorial notch. Jain $\mathrm{Vk}$ et $\mathrm{al}^{9}$ had all the cases operated by retro-sigmoid approach and concluded that the retro-sigmoid approach in experienced hands is a good option; with good results compared to other series irrespective of the tumor size. 
In giant VSs, total tumor removal can be achieved via the retrosigmoid approach without mortality and low morbidity, especially with regard to facial nerve function. ${ }^{11}$

\section{Postoperative outcomes}

CSF leakage was present in post operative in $23.07 \%$ $(\mathrm{n}=3)$ cases. This was managed conservatively with lumbar puncture. Merari $\mathrm{F}$ et al ${ }^{1}$ had rates of CSF leakage for retrosigmoid approach around $21 \%$. They found that tumor size and other factors such as delayed wound healing and episodes of increased intracranial pressure were important in CSF leakage. Gormley and Sekhar et al ${ }^{6}$ reported cerebrospinal fluid leakage in 15\% of the patient. The majority of the patients who experienced cerebrospinal fluid leakage were treated successfully with lumbar spinal drainage; only $2 \%$ (of the total group) required subsequent surgery for correction of cerebrospinal fluid leakage. Samii et $\mathrm{al}^{3}$ reported Cerebrospinal fluid leaks developed in 3 patients $(6 \%) 2$ were treated with lumbar drainage and 1 needed surgical closure. Jain $\mathrm{V} \mathrm{k}$ et al ${ }^{9} \mathrm{Cerebrospinal}$ fluid (CSF) leak occurred in 4\% patients. Most cases were managed conservatively with or without lumbar puncture and CSF drainage. Three patients required re-exploration and plugging of the drilled internal auditory meatus (IAM) for CSF leak.

Residual tumour was present in $2(15.38 \%)$ patients. Out of these 1 case belonged to Group 2 and 1 case belonged to group 3. Merari $\mathrm{F}$ et $\mathrm{al}^{1}$ had residual tumor in $7 \%$ for retrosigmoid approach Gormley and Sekhar et al ${ }^{6}$ reported Complete tumor resection was accomplished in $99 \%$ of the patients, and there was no evidence of recurrence in this group. Only 1 of the 179 patients underwent incomplete tumor resection; he required subsequent surgery for symptomatic tumor regrowth. Jain $\mathrm{Vk}$ et al ${ }^{9} \mathrm{had}$ achieved complete tumor excision was achieved in $96.5 \%$ cerebrospinal fluid (CSF) leak occurred in 4\% patients.

Post operative facial nerve functioning was assessed using House and Brackmann grading. ${ }^{7}$ Out of these Grade 1 functioning was present in $53.84 \%$ cases ( $n=7)$. Grade 3 and Grade 4 dysfunction were present in 2 patients. Grade 2 and grade 5 functions were present in 1 patient. Higher grades of House Bregman score $(>3)$ were present in $23.07 \%$ cases. In post operative follow up total 4 patients had symptomatic improvement. Out of them grade 2 and grade 4 function was present in 1 patient each and 2 patients had grade 3 dysfunction.

Merari $\mathrm{F}$ et $\mathrm{al}^{1}$ had reported Thirty-two patients (64\%) had a Grade 1 or 2 score at 1 year, while $26 \%$ had a score of 3 or 4 , and $8 \%$ had a score of 5 or 6 . They also found that there was a significant correlation between tumor size and facial nerve outcome, with larger tumors yielding worse outcomes. Gormley and Sekhar et $\mathrm{al}^{7}$ showed House-Brackmann evaluation of postoperative facial nerve function revealed excellent results (Grade I or II) in $96 \%$ of small tumors, $74 \%$ of medium tumors, and $38 \%$ of large tumors. A fair postoperative function (Grade III or IV) was achieved in $4 \%$ of small tumors, $26 \%$ of medium tumors, and $58 \%$ of large tumors. Samii et $\mathrm{al}^{3}$ in there series of 1000 cases showed that the facial nerve was anatomically preserved in 929 cases (93\%). In 200 cases of tumor resections using the CPA approach, preservation rates rose to $94 \%$, independent of tumor size. Facial nerve function graded according to the House-Brackmann scale within 2 weeks after surgery, was Grade 1 in $47 \%$, Grade 2 in $12 \%$, Grade 3 in 14\%, Grade 4 in $6 \%$, Grade 5 in $10 \%$, and Grade 6 in $11 \%$ of the patients.

Jain $\mathrm{V} \mathrm{k}$ et al ${ }^{9}$ in there series showed Facial nerve was anatomically preserved in $79.2 \%(198 / 250)$ patients with complete tumor excision.

Transient lower cranial nerve paresis was present in $46.15 \%$ $(\mathrm{n}=6)$ which gradually improved. Jain $\mathrm{V} \mathrm{k}$ et $\mathrm{al}^{9}$ had the incidence of lower cranial nerve paresis has been $6.8 \%$ in their study.

There was $1(7.6 \%)$ mortality which was due to post operative respiratory infection. Merari $\mathrm{F}$ et a ${ }^{1}$ had mortality of $2 \%$ for retrosigmoid approach. Gormley and Sekhar et al ${ }^{6} 1 \%$ mortality in there case series. Jain $\mathrm{V} k$ et al ${ }^{9}$ have observed mortality was $6 \%$ in their series. Sami $\mathrm{m}^{11}$ et al reported no mortality in their series.

\section{CONCLUSION}

The retromastoid approach can be utilized in excision of small, medium and large size acoustic schwanoma and prior to undertaking a complete excision a Ventriculo-peritoneal shunting can be done on emergency basis to counteract the raised intracranial pressure. Combination of above two can help in bringing good neurological outcome in patients with CPA tumors.

\section{REFERENCES}

1. Memari F, Hassannia F and Abtahi SHR. Surgical Outcomes of Cerebellopontine angle Tumors in 50 Cases. Iranian Journal of Otorhinolaryngology 2015; 27(78):29-34.

2. Iwai $\mathrm{Y}$, Yamanka $\mathrm{K}$ and Ishiguro $\mathrm{T}$. Surgery combined with radiosurgery of large acoustic neuromas. Surg Neurol 2003; 59(4):283-291.

3. Samii $M$ and Matthies C. Management of 1000 vestibular schwannomas (acoustic neuromas): Surgical management and results with an emphasis on complications and how to avoid them. Neurosurgery 1997; 40:11-23. 
4. Ramamurthi $B$. The continuing challenge of acoustic neurinomas (1949-1993). Br J Neurosurg 1995; 9:361-366.

5. Madjid S, Hussam M, Amir S and Venelin G. Retrosigmoid Intradural Inframeatal Approach: Indications and Technique. Neurosurgery 2013; 73: 53.60 .

6. Gormley WB, Sekhar LN, Wright DC, Kamerer D and Schessel D. Acoustic neuromas. Results of current surgical management. Neurosurgery 1997; 41:50-60.

7. House JW and Brackmann DE. Facial nerve grading system. Otolaryngol Head Neck Surg1985; 93(2):146-147.

8. Mirzayan MJ, Gerganov VM, Lüdemann W, Oi S, Samii M and Samii A. Management of vestibular schwannomas in young patients-comparison of clinical features and outcome with adult patients. Child's Nervous System 23:8, 891-895.

9. Jain VK, Mehrotra N, Sahu RN, Behari S, Banerji D and Chhabra DK. Surgery of vestibular schwannomas: An institutional experience. Neurol India 2005; 53:41-45.

10. Gerganov VM, Pirayesh A, Nouri M, Hore N, Luedemann WO and Oi S. Hydrocephalus associated with vestibular schwannomas: management options and factors predicting the outcome. J Neurosurg 2011; 114:1209-1215.

11. Samii M, Gerganov VM and Samii A. Functional outcome after complete surgical removal of giant vestibular schwannomas. J Neurosurg 2010;112(4):860-867.

\section{Authors Contribution:}

SD - Assistant surgeon, Concept and design of the study, reviewed the literature manuscript preparation and revision of the manuscript; MSB - Assistant surgeon, Concept and design of the study, helped in preparing first draft of manuscript and revision of the manuscript; SKS - Main operating surgeon, Concept and design of the study, manuscript preparation and revision of the manuscript; KR - Main operating surgeon, manuscript preparation and revision of the manuscript; PG - Assistant surgeon Conceptualized study, literature search, statistically analyzed and interpreted; SBV - Assistant surgeon, Conceptualized study, literature search, collected data and review of study.

Orcid ID:

Dr. Sourabh Dixit:(i) https://orcid.org/0000-0003-1085-7000

Source of Support: None. Conflict of interest: None declared. 